\title{
Rückblick auf das 20. Fortbildungsseminar der APT in Magdeburg
}

Auch in diesem Jahr fand zwischen Röntgenkongress und Sommer das Fortbildungsseminar der AG Physik und Technik in der bildgebenden Diagnostik (APT)

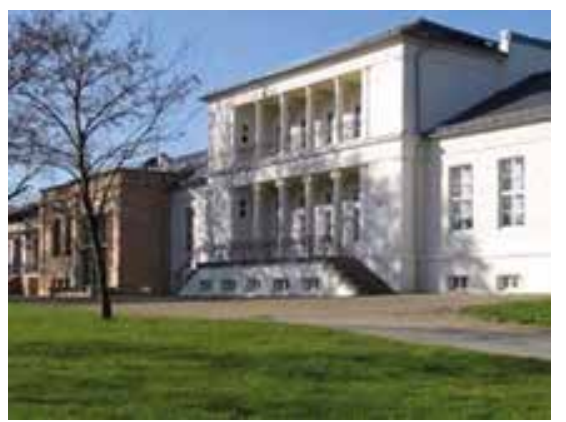

statt. Diesmal jährte es sich zum 20. Male. Medizinphysiker, Radiologen, MTRAs, Sachverständige und andere interessierte Teilnehmer trafen sich am 10. und 11. Juni 2016 im Gesellschaftshaus am Klosterbergegarten in Magdeburg.

Das Seminar begann mit einem kurzen Rückblick des Vorstandes auf das 20-jährige Bestehen der APT und ließ die Anfänge der APT innerhalb der Deutschen Röntgengesellschaft Revue passieren. Schwerpunkt des ersten Tags war anschließend die Magnetresonanztomografie. Vorträge zur Sicherheit bei der MRT, zum Hochfeld-MRT und zum

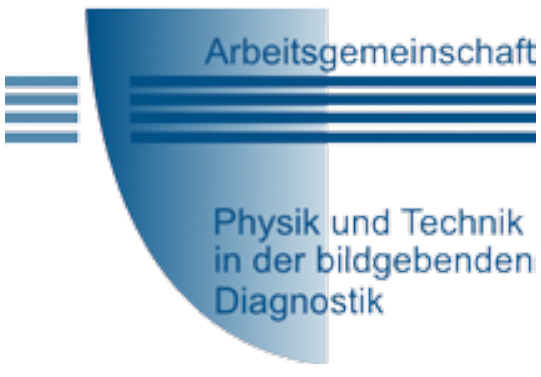

Umgang mit Herzschrittmacher-Patienten wurden präsentiert, wobei speziell auf das aktuelle Positionspapier der DRG (Fortschr Röntgenstr 2015; 187: 777-787) eingegangen wurde. Natürlich durften die praktischen Aspekte des Strahlenschutzes beim APT-Seminar nicht fehlen. Vor dem Hintergrund der bevorstehenden Reduzierung des Grenzwerts für die Augenlinse lag ein Schwerpunkt auf der Personendosimetrie, ein weiterer auf den speziellen Anforderungen in der Kinderradiologie. 

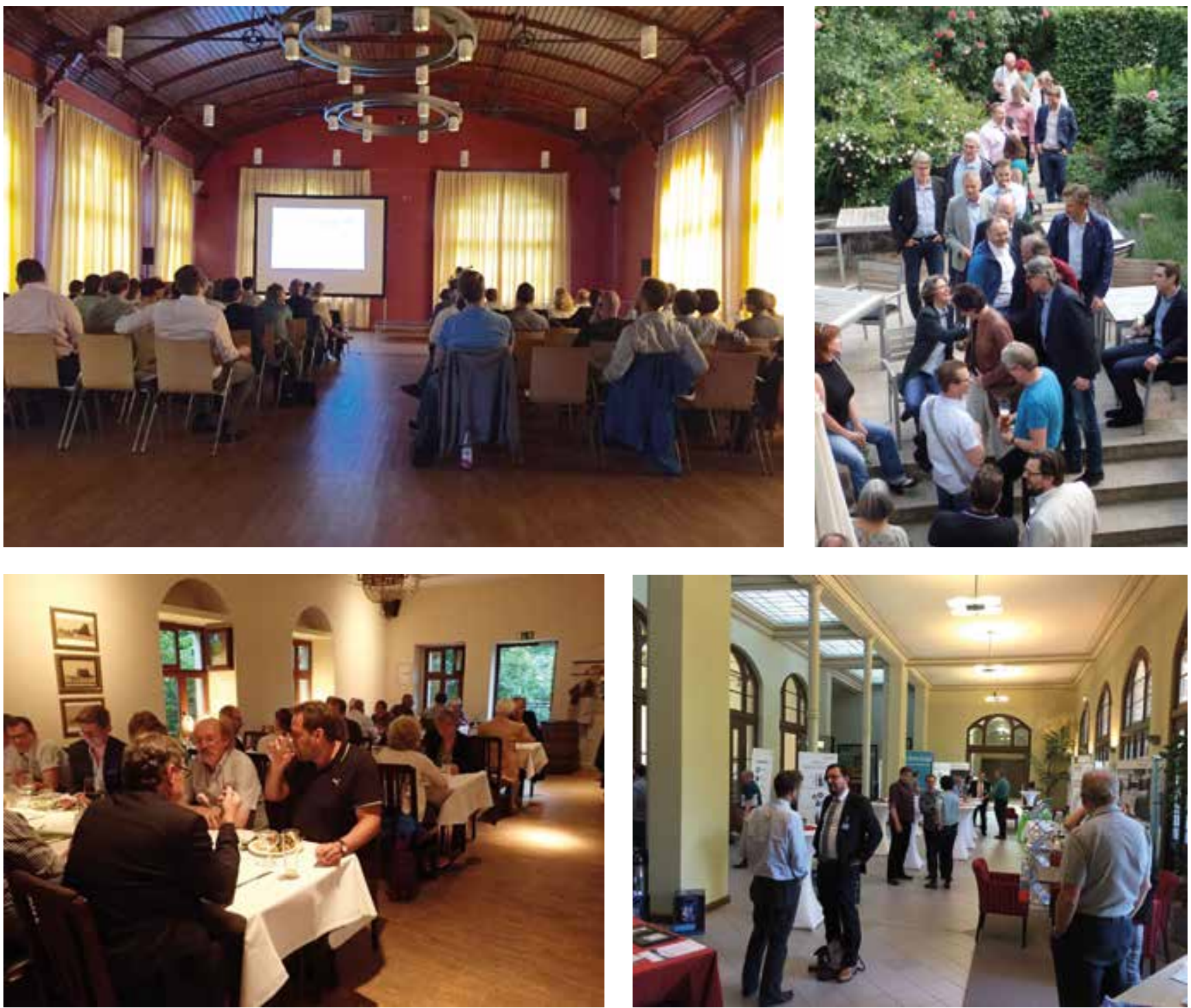

Für den individuellen Erfahrungsaustausch war am Abend genügend Zeit, wobei die Rahmenbedingungen dafür im zwischen Dom und Elbe gelegenen „Hoflieferanten“ bestens geeignet waren.

Der zweite Tag begann mit Vorträgen zur Optimierung des Strahlenschutzes bei Interventionen, wobei das Augenmerk auf die Reduzierung der Dosis von Patient und Personal gerichtet wurde. Dies wurde den interessierten Teilnehmern am Beispiel von Interventionen in der Angiografie, im Hybrid-OP und im CT nahe gebracht. Die nachfolgende Sitzung infor- mierte über neue Normen und Richtlinien einschließlich der neuen Diagnostischen Referenzwerte und schloss mit einer lebhaften Diskussionsrunde über den Medizin-Physik-Experten nach RöV, der in der neuen Strahlenschutzgesetzgebung 2017 verpflichtend eingeführt wird. Die letzte Sitzung des Tages wurde gemeinsam mit der AG Informationstechnologie (@GIT) zum Thema PACS durchgeführt. Damit wurde ein neues Format (APT meets ...) gestartet, in dem die APT zukünftig die Zusammenarbeit mit anderen AGs der DRG intensivieren möchte.
Die gelungene, interessante und lebhafte Veranstaltung zu aktuellen Themen fand in äußerst angenehmer Atmosphäre statt und ließ ausreichend Zeit für Diskussionen und Gesprächen mit Kollegen. Ausdrücklich erwähnt sei die professionelle Begleitung durch die Geschäftsstelle der DRG, die sehr zum Gelingen des Seminars beigetragen hat.

\section{Dr. Kerstin Jungnickel, Vorstand der APT}

